\title{
CHARACTERIZATIONS OF METRIC TREES AND GROMOV HYPERBOLIC SPACES
}

\author{
Stefan Wenger
}

\begin{abstract}
In this note we give new characterizations of metric trees and Gromov hyperbolic spaces and generalize recent results of Chatterji and Niblo. Our results have a purely metric character, however, their proofs involve two classical tools from analysis: Stokes' formula in $\mathbb{R}^{2}$ and a Rademacher type differentiation theorem for Lipschitz maps.
\end{abstract}

\section{Statement of the main results}

A geodesic metric space is said to be a metric tree (or an $\mathbb{R}$-tree) if it is 0 -hyperbolic in the sense of Gromov, or in other words, if all its geodesic triangles are isometric to tripods. Metric trees are used in geometry, metric topology, geometric group theory and also in the geometry of Banach spaces (see e.g. [8] for the latter). They have the following property which can easily be verified (see Lemma 3.7): The intersection $B_{1} \cap B_{2}$ of any two closed balls $B_{1}, B_{2}$ is a ball or the empty set. In this note we prove that this property already characterizes metric trees and that, maybe somewhat surprisingly, already a weaker property for a geodesic metric space implies that it is a metric tree. Namely, we show:

Theorem 1.1. Let $(X, d)$ be a geodesic metric space and $\lambda \geq 1$. If for any two balls $B_{1}, B_{2} \subset X$ with non-empty intersection there exist $z, z^{\prime} \in X$ and $\nu \geq 0$ such that

$$
B(z, \nu) \subset B_{1} \cap B_{2} \subset B\left(z^{\prime}, \lambda \nu\right)
$$

then $X$ is a metric tree.

Here, $B(z, \nu):=\{x \in X: d(x, z) \leq \nu\}$. As a special case we obtain the following characterization of metric trees, initially conjectured by Chatterji-Niblo and, shortly after a preliminary version of our paper had appeared, established independently in [3] with metric methods:

Corollary 1.2. Let $(X, d)$ be a geodesic metric space. Then $X$ is a metric tree if and only if the intersection of any two closed balls is a ball or the empty set.

As another application of Theorem 1.1 we obtain a generalization of the main result of $[3]$.

Corollary 1.3. Let $(X, d)$ be a geodesic metric space and suppose there exist $\lambda, \delta>0$ with the following property: For any two balls $B_{1}, B_{2} \subset X$ with non-empty intersection there exist $z, z^{\prime} \in X$ and $r \geq 0$ such that

$$
B(z, r) \subset B_{1} \cap B_{2} \subset B\left(z^{\prime}, \lambda r+\delta\right) .
$$

Received by the editors August 26, 2007. 
Then $X$ is Gromov hyperbolic.

Conversely, if $X$ is a geodesic Gromov hyperbolic space then it is not difficult to show that (1) holds with $\lambda=1$ and some $\delta$ only depending on the constant of Gromov hyperbolicity. For a proof of this see Lemma 3.7 or [3]. For the definition of Gromov hyperbolicity see Section 2.

We note that in general, Theorem 1.1 is false if $X$ is not geodesic. Indeed, if $X:=$ $\left(\mathbb{R},|x-y|^{\frac{1}{2}}\right)$ then the intersection of any two closed balls in $X$ is either a ball or the empty set. However, there does not exist a bilipschitz embedding of $X$ into a metric tree.

A remarkable feature of our note is the analytic flavor of our proof of the purely metric statement of Theorem 1.1. A similar approach is used in [11] to give a characterization of geodesic Gromov hyperbolic spaces in terms of a quadratic isoperimetric inequality with the sharp isoperimetric constant.

1.1. Outline of the proof of Theorem 1.1. The starting point of the proof of our main theorem is the following simple but useful observation which provides yet another new characterization of metric trees.

Proposition. A geodesic metric space $X$ is a metric tree if and only if for every Lipschitz loop $\gamma: S^{1} \rightarrow X$ and all Lipschitz functions $f, \pi: X \rightarrow \mathbb{R}$

$$
\int_{S^{1}}(f \circ \gamma)(t) \cdot(\pi \circ \gamma)^{\prime}(t) d t=0
$$

This proposition will be proved in Section 3 and will be used as follows: Let $X$ satisfy the hypotheses of Theorem 1.1. In Lemma 3.3 and Corollary 3.5 we first show that $X$ is Lipschitz 1-connected, i.e., that for every Lipschitz loop $\gamma: S^{1} \rightarrow X$ there exists a Lipschitz extension $\tilde{\gamma}: B^{2} \rightarrow X$ to the unit disc $B^{2} \subset \mathbb{R}^{2}$. Next, we prove in Lemma 3.6 that

$$
\operatorname{det}\left(D_{z}[(f, \pi) \circ \tilde{\gamma}]\right)=0 \quad \text { for almost every } z \in B^{2},
$$

for all Lipschitz functions $f, \pi: X \rightarrow \mathbb{R}$. Here, $D_{z}[(f, \pi) \circ \tilde{\gamma}]$ denotes the classical derivative at $z$, which exists almost everywhere by Rademacher's theorem. Roughly, (3) holds because otherwise, $X$ would contain almost-isometric copies of pieces of a 2 -dimensional normed space by a generalized Rademacher theorem. This however is easily seen to contradict the assumption on the intersection of balls. Finally, Stokes' theorem together with (3) yields (2), implying that $X$ is a metric tree. The generalized Rademacher theorem alluded to above is due to Kirchheim and independently to Korevaar-Schoen. In the appendix we will give a short and partially new proof of this theorem.

\section{Preliminaries}

2.1. Geodesic metric spaces, metric trees and Gromov hyperbolicity. Let $(X, d)$ be a metric space and $a>0$. A map $c:[0, a] \rightarrow X$ is called a (constant speed) geodesic path from $c(0)$ to $c(a)$ if there exists $\lambda>0$ such that $d(c(s), c(t))=\lambda|s-t|$ for all $s, t \in[0, a]$. If $\lambda=1$ then $c$ is called a geodesic path parameterized by arc-length. The metric space $X$ is called geodesic if any two points can be joined by a geodesic path. 
A geodesic triangle in $X$ consists of three points (the vertices) in $X$ and a choice of three geodesics (the edges) joining these points. Let $\Delta$ be a geodesic triangle and $\delta \geq 0$. Denote by $x_{1}, x_{2}, x_{3} \in X$ its vertices and by $c_{k \ell}$ its edges (joining $x_{k}$ to $x_{\ell}$ ), which we may assume to be parameterized by arc-length, $1 \leq k<\ell \leq 3$. There exist unique numbers $a_{1}, a_{2}, a_{3} \geq 0$ with the property that $d\left(x_{k}, x_{\ell}\right)=a_{k}+a_{\ell}$. If $1 \leq k<\ell \leq 3$ we define $c_{\ell k}:\left[0, a_{k}+a_{\ell}\right] \rightarrow X$ by $c_{\ell k}(t):=c_{k \ell}\left(a_{k}+a_{\ell}-t\right)$. Then $\Delta$ is said to be $\delta$-thin if for every permutation $\sigma$ of $\{1,2,3\}$

$$
d\left(c_{\sigma(1) \sigma(2)}(t), c_{\sigma(1) \sigma(3)}(t)\right) \leq \delta
$$

for all $t \in\left[0, a_{\sigma(1)}\right]$.

Definition 2.1. A geodesic metric space $(X, d)$ is called Gromov hyperbolic if there exists $\delta \geq 0$ such that every geodesic triangle in $X$ is $\delta$-thin. If $\delta=0$ then $X$ is called a metric tree.

Gromov hyperbolic spaces were introduced and first studied by Gromov in [7] in the context of geometric group theory. See for example [2] or [6] for a treatment of the topic.

2.2. Lipschitz maps and metric derivatives. The following notion of differentiability for metric space valued Lipschitz maps was introduced by Kirchheim in [9]. Let $\varphi: U \rightarrow X$ be a Lipschitz map, where $U \subset \mathbb{R}^{k}$ is open and $(X, d)$ a metric space. The metric directional derivative of $\varphi$ at $z \in U$ in direction $v \in \mathbb{R}^{k}$ is defined by

$$
\operatorname{md} \varphi_{z}(v):=\lim _{r \searrow 0} \frac{d(\varphi(z+r v), \varphi(z))}{r}
$$

if this limit exists. We will make use of the following trivial remark in the proof of our main theorem.

Remark 2.2. Let $\varphi: U \subset \mathbb{R}^{k} \rightarrow X$ and $\varrho: X \rightarrow \mathbb{R}^{k}$ be Lipschitz maps and $z \in U$. If $\varrho \circ \varphi$ is differentiable at $z$ and $\operatorname{md} \varphi_{z}(v)$ exists for all $v \in \mathbb{R}^{k}$ and is degenerate (that is $\operatorname{md} \varphi_{z}(v)=0$ for some $\left.v \neq 0\right)$, then

$$
\operatorname{det}\left(D_{z}[\varrho \circ \varphi]\right)=0 .
$$

The following theorem was proved by Kirchheim in [9] and a similar statement by Korevaar-Schoen in [10]. We will provide a partially new proof in the appendix.

Theorem 2.3. Let $(X, d)$ be a metric space and $\varphi: U \rightarrow X$ a Lipschitz map, where $U \subset \mathbb{R}^{k}$ is open. Then for almost every $z \in U$ the metric directional derivative $\operatorname{md} \varphi_{z}(v)$ exists for every $v \in \mathbb{R}^{k}$. Furthermore, there are compact sets $K_{i} \subset U$, $i \in \mathbb{N}$, such that $\mathcal{L}^{k}\left(U \backslash \cup K_{i}\right)=0$ and such that the following property holds: For every $i$ and every $\varepsilon>0$ there exists $r\left(K_{i}, \varepsilon\right)>0$ such that

$$
\left|d(\varphi(z+v), \varphi(z+w))-\operatorname{md} \varphi_{z}(v-w)\right| \leq \varepsilon|v-w|
$$

for all $z \in K_{i}$ and all $v, w \in \mathbb{R}^{k}$ satisfying $|v|,|w| \leq r\left(K_{i}, \varepsilon\right)$ and $z+w \in K_{i}$.

Here $|\cdot|$ denotes the Euclidean norm and $\mathcal{L}^{k}$ the Lebesgue measure. If $\operatorname{md} \varphi_{z}(v)$ exists for all $v \in \mathbb{R}^{k}$ and satisfies (4) then $\operatorname{md} \varphi_{z}$ is called metric derivative of $\varphi$ at the point $z$. As a direct consequence of Theorem 2.3 the metric derivative is a seminorm. It is not difficult to prove that if $U \subset \mathbb{R}^{k}$ is merely Borel measurable then md $\varphi_{z}$ can 
be defined at almost every Lebesgue density point $z \in U$ by a simple approximation argument.

\section{Proof of Theorem 1.1}

The following characterization of metric trees will be used in the proof of Theorem 1.1.

Proposition 3.1. Let $X$ be a geodesic metric space. If for every Lipschitz curve $\gamma:[0,1] \rightarrow X$ with $\gamma(0)=\gamma(1)$ and all Lipschitz functions $f, \pi: X \rightarrow \mathbb{R}$

$$
\int_{0}^{1}(f \circ \gamma)(t) \cdot(\pi \circ \gamma)^{\prime}(t) d t=0
$$

then $X$ is a metric tree.

Note that the function $\pi \circ \gamma$ is a Lipschitz function on $[0,1]$ and therefore, by Rademacher's theorem, its classical derivative $(\pi \circ \gamma)^{\prime}(t)$ exists for almost every $t \in[0,1]$ and defines a bounded Borel function. In particular, the integral in (5) is well-defined.

Proof. We first show that $X$ is uniquely geodesic. For this let $\gamma_{1}, \gamma_{2}:[0,1] \rightarrow X$ be two geodesics with the same endpoints. Set $a:=d\left(\gamma_{1}(0), \gamma_{1}(1)\right)$ and define a Lipschitz map by $\pi(x):=d\left(x, \gamma_{1}(0)\right)$ and let $\gamma:[0,1] \rightarrow X$ be the constant speed loop given by the concatenation of $\gamma_{1}$ and $\gamma_{2}^{-}(t):=\gamma_{2}(1-t)$. Then $(\pi \circ \gamma)^{\prime}(t)=2 a$ for every $t \in\left[0, \frac{1}{2}\right]$ and $(\pi \circ \gamma)^{\prime}(t)=-2 a$ for every $t \in\left[\frac{1}{2}, 1\right]$. Setting $f(x):=\max \left\{0,1-\operatorname{dist}\left(\gamma_{1}([0,1]), x\right)\right\}$ it follows immediately from (5) that $\gamma_{2}([0,1]) \subset \gamma_{1}([0,1])$ and therefore that $\gamma_{2}(t)=$ $\gamma_{1}(t)$ for all $t \in[0,1]$.

We now prove that every geodesic triangle in $X$ is a tripod. For this let $\gamma_{i}:\left[0, a_{i}\right] \rightarrow X$, $i=1,2,3$, be three geodesics parameterized by arc-length forming a geodesic triangle, i.e., such that $\gamma_{1}\left(a_{1}\right)=\gamma_{2}(0), \gamma_{2}\left(a_{2}\right)=\gamma_{3}(0)$, and $\gamma_{3}\left(a_{3}\right)=\gamma_{1}(0)$. Set

$$
\begin{aligned}
& t_{1}:=\max \left\{t \in\left[0, a_{1}\right]: \gamma_{1}(t) \in \gamma_{3}\left(\left[0, a_{3}\right]\right)\right\} \\
& t_{2}:=\min \left\{t \in\left[0, a_{1}\right]: \gamma_{1}(t) \in \gamma_{2}\left(\left[0, a_{2}\right]\right)\right\} .
\end{aligned}
$$

By defining $\gamma:[0,1] \rightarrow X$ to be the constant speed loop given by the concatenation of $\gamma_{1}, \gamma_{2}$ and $\gamma_{3}$ and by setting $\pi(x):=d\left(x, \gamma_{1}(0)\right)$ and

$$
f_{\varepsilon}(x):=\max \left\{0,1-\varepsilon^{-1} \operatorname{dist}\left(x, \gamma_{1}([0,1])\right)\right\}
$$

we easily find using the uniqueness of geodesics and the fact that

$$
\int_{0}^{1}\left(f_{\varepsilon} \circ \gamma\right)(t) \cdot(\pi \circ \gamma)^{\prime}(t) d t=0
$$

for every $\varepsilon>0$ that $t_{1}=t_{2}$ and hence $\gamma_{1}\left(t_{1}\right) \in \operatorname{Im}\left(\gamma_{2}\right) \cap \operatorname{Im}\left(\gamma_{3}\right)$. By the uniqueness of geodesics the triangle consisting of $\gamma_{1}, \gamma_{2}, \gamma_{3}$ is thus a tripod. This completes the proof.

The proof of Theorem 1.1 involves several other auxiliary results which we state now. In order to simplify the language in what follows it is convenient to introduce a new terminology. We say a metric space $X$ has property $(\diamond)$ if it satisfies the hypotheses of Theorem 1.1, that is, if there exists $\lambda \geq 1$ such that for any two closed balls $B_{1}, B_{2} \subset X$ with non-empty intersection there exist $z, z^{\prime} \in X$ and $\nu \geq 0$ such that

$$
B(z, \nu) \subset B_{1} \cap B_{2} \subset B\left(z^{\prime}, \lambda \nu\right) .
$$


To prove Theorem 1.1 it will in fact be enough to require (6) only for those balls $B_{1}=B(x, r)$ and $B_{2}=B\left(x^{\prime}, r^{\prime}\right)$ for which $\max \left\{r, r^{\prime}\right\}<d\left(x, x^{\prime}\right)$. It is then readily verified that (6) can be replaced by the hypothesis

$$
\operatorname{diam}\left(B(x, r) \cap B\left(x^{\prime}, r^{\prime}\right)\right) \leq 2 \lambda\left(r+r^{\prime}-d\left(x, x^{\prime}\right)\right) .
$$

We begin with the following observation.

Remark 3.2. Let $X$ be a geodesic metric space with property $(\diamond)$. Then for any two distinct points $x, y \in X$ and for all $t \in(0,1)$ and $0 \leq h<\max \{t, 1-t\} d(x, y)$ the set

$$
A:=B(x, t r+h) \cap B(y,(1-t) r+h),
$$

where $r:=d(x, y)$, satisfies $\operatorname{diam} A \leq 4 \lambda h$.

Choosing $h=0$ in the remark we obtain that such a space $X$ is uniquely geodesic.

Proof of Remark 3.2. By symmetry we may assume that $t \leq \frac{1}{2}$. Note that $A$ is nonempty since $X$ is geodesic; moreover $x, y \notin A$. By assumption there exist $z, z^{\prime} \in X$ and $s \geq 0$ such that $B(z, s) \subset A \subset B\left(z^{\prime}, \lambda s\right)$. It is clear that $s<d(x, z)$. Let $\alpha$ be a geodesic from $z$ to $x$ parameterized by arc-length. Then $\alpha(s) \in A$ and hence

$$
r \leq d(x, \alpha(s))+d(\alpha(s), y) \leq t r+h-s+(1-t) r+h
$$

from which it follows that $s \leq 2 h$ and hence $\operatorname{diam} A \leq 2 \lambda s \leq 4 \lambda h$.

Lemma 3.3. If $X$ is a geodesic metric space with property $(\diamond)$ then for any points $x, y, y^{\prime} \in X$ the geodesics $c, c^{\prime}:[0,1] \rightarrow X$, parameterized proportional to arc-length, with $c(0)=x=c^{\prime}(0)$ and $c(1)=y$ and $c^{\prime}(1)=y^{\prime}$ satisfy

$$
d\left(c(t), c^{\prime}(t)\right) \leq 4 \lambda d\left(y, y^{\prime}\right)
$$

for all $t \in[0,1]$.

Proof. Let $x, y, y^{\prime} \in X$ be arbitrary points and set $r:=d(x, y)$ and $r^{\prime}:=d\left(x, y^{\prime}\right)$. If $y=x$ or $y^{\prime}=x$ then the statement is trivially true and we may therefore assume that $0<r^{\prime} \leq r$. Set $h:=d\left(y, y^{\prime}\right)$ and fix $t \in(0,1)$. If $h \geq t r$ then

$$
d\left(c(t), c^{\prime}(t)\right) \leq d(c(t), x)+d\left(x, c^{\prime}(t)\right)=t r+t r^{\prime} \leq 2 h .
$$

If, on the other hand, $h<t r$ then we define

$$
A:=B(x, t r+h) \cap B(y,(1-t) r+h)
$$

and obtain that

$$
c^{\prime}(t) \in B\left(x, t r^{\prime}\right) \cap B\left(y^{\prime},(1-t) r^{\prime}\right) \subset B(x, t r) \cap B(y,(1-t) r+h) \subset A
$$

as well as $c(t) \in A$. Since by Remark 3.2 we have $\operatorname{diam} A \leq 4 \lambda h$ it follows that

$$
d\left(c(t), c^{\prime}(t)\right) \leq 4 \lambda d\left(y, y^{\prime}\right) \text {. }
$$

This concludes the proof.

Remark 3.4. It should be noted that the assertions of Remark 3.2 and Lemma 3.3 remain true if $(\diamond)$ is replaced by the following condition: For any two balls $B(x, r), B(y, s) \subset X$ with non-empty intersection and every $\varepsilon>0$ there exist $z, z^{\prime} \in X$ and $t \geq 0$ with

$$
B(z, t-\varepsilon) \subset B(x, r+\varepsilon) \cap B(y, s+\varepsilon)
$$


and

$$
B(x, r) \cap B(y, s) \subset B\left(z^{\prime}, \lambda(t+\varepsilon)\right) .
$$

This property will be called $\left(\diamond^{\prime}\right)$ and will be used in the proof of Corollary 1.3.

As a consequence of the lemma above we obtain the following extension property.

Corollary 3.5. If $X$ is a geodesic metric space with property $\left(\triangleright^{\prime}\right)$ then every Lipschitz map $\varphi: S^{m} \rightarrow X$ has a Lipschitz extension $\bar{\varphi}: B^{m+1} \rightarrow X$ with $\operatorname{Lip}(\bar{\varphi}) \leq(8 \lambda+$ 12) $\operatorname{Lip}(\varphi)$. In other words, $X$ is Lipschitz $m$-connected for every $m \geq 0$.

Here, $S^{m}$ and $B^{m+1}$ denote the unit sphere and unit ball of $\mathbb{R}^{m+1}$ with the Euclidean metric, respectively.

Proof. For a given Lipschitz map $\varphi: S^{m} \rightarrow X$ fix $x_{0} \in \varphi\left(S^{m}\right)$ and define $\bar{\varphi}: B^{m+1} \rightarrow$ $X$ by

$$
\bar{\varphi}(r z):=\left\{\begin{array}{lll}
x_{0} & : & 0 \leq r \leq \frac{1}{2} \\
c_{z}\left(2\left(r-\frac{1}{2}\right)\right) & : & \frac{1}{2}<r \leq 1
\end{array}\right.
$$

where $c_{z}:[0,1] \rightarrow X$ denotes the constant-speed geodesic from $x_{0}$ to $\varphi(z)$. This clearly defines a Lipschitz map which extends $\varphi$. A simple calculation using Lemma 3.3 shows furthermore that $\operatorname{Lip}(\bar{\varphi}) \leq(8 \lambda+12) \operatorname{Lip}(\varphi)$.

In the proof of the following lemma we will use the obvious but useful fact that the properties $(\diamond)$ and $\left(\diamond^{\prime}\right)$ persist under rescaling of the metric.

Lemma 3.6. Let $X$ be a geodesic metric space with property $\left(\diamond^{\prime}\right)$ and let $K \subset \mathbb{R}^{2}$ be Borel measurable. If $\varphi: K \rightarrow X$ is a Lipschitz map then $\operatorname{md} \varphi_{z}$ is degenerate for almost all $z \in K$.

Proof. We argue by contradiction and assume that $\mathrm{md} \varphi_{z}$ is non-degenerate, and thus a norm, for all $z$ in some set of positive Lebesgue measure. By Theorem 2.3 there exists a compact set $K^{\prime} \subset K$ of positive Lebesgue measure and a Lebesgue density point $z_{0} \in K^{\prime}$ such that the norm $\|\cdot\|:=\operatorname{md} \varphi_{z_{0}}$ has the following property. For every $\varepsilon>0$ there exists $r_{0}>0$ such that the map $\tilde{\varphi}:\left(K^{\prime}-z_{0},\|\cdot\|\right) \rightarrow X$ given by $\tilde{\varphi}(v):=\varphi\left(z_{0}+v\right)$ is $(1+\varepsilon)$-bilipschitz on $B\left(0, r_{0}\right) \cap\left(K^{\prime}-z_{0}\right)$. Here, $B\left(0, r_{0}\right)$ denotes the ball of radius $r_{0}$ with respect to the Euclidean metric whereas we will denote by $\hat{B}(0, r)$ the ball of radius $r$ with respect to $\|\cdot\|$. Note that $\hat{B}(0,1)$ is convex, centrally symmetric and closed with respect to the Euclidean metric. Let $B\left(0, r_{1}\right) \subset \hat{B}(0,1)$ be the Euclidean ball of maximal radius and let $v_{0} \in \partial B\left(0, r_{1}\right) \cap \partial \hat{B}(0,1)$. Set $y:=2 v_{0}$. It is then easy to see that

$$
\frac{1}{h} \operatorname{diam}_{\|\cdot\|}[\hat{B}(0,1+h) \cap \hat{B}(y, 1+h)] \rightarrow \infty \quad \text { as } h \rightarrow 0 .
$$

Indeed, this follows from the fact that

$$
\hat{B}(0,1+h) \cap \hat{B}(y, 1+h) \supset B\left(0, r_{1}(1+h)\right) \cap B\left(y, r_{1}(1+h)\right)
$$

and

$$
\operatorname{diam}_{\text {eucl. }}\left[B\left(0, r_{1}(1+h)\right) \cap B\left(y, r_{1}(1+h)\right)\right]=2 \sqrt{2 h+h^{2}} r_{1} .
$$


Since $z_{0}$ is a Lebesgue density point of $K^{\prime}$ there exist sequences $r_{n} \searrow 0$ and $y_{n} \in \mathbb{R}^{2}$ such that $y_{n} \in K_{n}:=\frac{1}{r_{n}}\left(K^{\prime}-z_{0}\right)$ and $s_{n}:=\left\|y_{n}-y\right\| \rightarrow 0$ and such that for every $h>0$

$$
\left[\hat{B}(0,1+h) \cap \hat{B}\left(y_{n}, 1+h\right) \cap K_{n}\right] \longrightarrow[\hat{B}(0,1+h) \cap \hat{B}(y, 1+h)]
$$

with respect to Hausdorff convergence. Denote by $X_{n}$ the metric space $\left(X, r_{n}^{-1} d\right)$ and note that $X_{n}$ has property $\left(\diamond^{\prime}\right)$. Then the maps $\tilde{\varphi}_{n}:\left(K_{n},\|\cdot\|\right) \rightarrow X_{n}$ given by $\tilde{\varphi}_{n}(u):=\tilde{\varphi}\left(r_{n} u\right)$ are $\left(1+\varepsilon_{n}\right)$-bilipschitz with $\varepsilon_{n} \searrow 0$, see Theorem 2.3. Remark 3.2 then implies that for $h>0$ small enough

$$
\operatorname{diam}_{X_{n}}\left[B_{X_{n}}\left(\tilde{\varphi}_{n}(0), t_{n}+h\right) \cap B_{X_{n}}\left(\tilde{\varphi}_{n}\left(y_{n}\right), t_{n}+h\right)\right] \leq 4 \lambda h
$$

for every $n \in \mathbb{N}$, where $t_{n}:=\frac{1}{2} d_{X_{n}}\left(\tilde{\varphi}_{n}(0), \tilde{\varphi}_{n}\left(y_{n}\right)\right)$. On the other hand, it follows from (8) that for $n$ large enough

$$
\begin{aligned}
\operatorname{diam}_{X_{n}} & {\left[B_{X_{n}}\left(\tilde{\varphi}_{n}(0), t_{n}+h\right) \cap B_{X_{n}}\left(\tilde{\varphi}_{n}\left(y_{n}\right), t_{n}+h\right)\right] } \\
\geq & \frac{1}{1+\varepsilon_{n}} \operatorname{diam}_{\|\cdot\|}\left[\hat{B}\left(0,\left(1+\varepsilon_{n}\right)^{-1}\left(t_{n}+h\right)\right) \cap \hat{B}\left(y_{n},\left(1+\varepsilon_{n}\right)^{-1}\left(t_{n}+h\right)\right) \cap K_{n}\right] \\
\geq & \frac{1}{2\left(1+\varepsilon_{n}\right)} \operatorname{diam}_{\|\cdot\|}\left[\hat{B}\left(0,1+h_{n}^{\prime}\right) \cap \hat{B}\left(y_{n}, 1+h_{n}^{\prime}\right)\right]
\end{aligned}
$$

where

$$
h_{n}^{\prime}=\frac{h-\frac{s_{n}}{2}-2 \varepsilon_{n}-\varepsilon_{n}^{2}}{\left(1+\varepsilon_{n}\right)^{2}} .
$$

Using the fact that $\varepsilon_{n}, s_{n} \rightarrow 0^{+}$together with (7) we readily arrive at a contradiction with (9). This completes the proof.

We are finally ready to prove Theorem 1.1.

Proof of Theorem 1.1. We first show that if $X$ has property $\left(\diamond^{\prime}\right)$ then $X$ is a metric tree. We do this by verifying the hypothesis (5) of Proposition 3.1. For this let $\gamma: S^{1} \rightarrow X$ be a Lipschitz loop and let $\tilde{\gamma}: B^{2} \rightarrow X$ be a Lipschitz extension, which exists by Corollary 3.5. Let $f, \pi: X \rightarrow \mathbb{R}$ be arbitrary Lipschitz functions. By Stokes' Theorem and an obvious smoothing argument we obtain

$$
\int_{S^{1}}(f \circ \gamma)(t) \cdot(\pi \circ \gamma)^{\prime}(t) d t=\int_{B^{2}} \operatorname{det}\left(D_{z}[(f, \pi) \circ \tilde{\gamma}]\right) d z=0,
$$

where the second equality follows from Lemma 3.6 and Remark 2.2. It now follows from Proposition 3.1 that $X$ is a metric tree. The proof of the converse statement is a consequence of the lemma below with $\delta=0$.

Lemma 3.7. Let $\delta \geq 0$ and suppose $(X, d)$ is a geodesic metric space all of whose geodesic triangles are $\delta$-thin. Then for any two closed balls $B_{1}, B_{2}$ in $X$ with nonempty intersection there exist $z \in X$ and $\nu \geq 0$ such that

$$
B(z, \nu) \subset B_{1} \cap B_{2} \subset B(z, \nu+\delta) .
$$

The proof of the lemma is straight-forward and is implicitly contained in [3]. For completeness we give our own short proof here. 
Proof. Let $B(x, r)$ and $B(y, s)$ be two closed balls in $X$ and suppose $K:=B(x, r) \cap$ $B(y, s)$ is non-empty. We may assume without loss of generality that $r \geq s$. Set $d:=d(x, y)$. If $r-s>d$ then it follows that $K=B(y, s)$ and there is nothing to prove. If $r-s \leq d$ then choose a geodesic $c$ between $x$ and $y$ and let $z \in \operatorname{Im}(c)$ be such that

$$
d(x, z)=\frac{r-s+d}{2} \text { and } d(y, z)=\frac{s-r+d}{2} .
$$

Set furthermore $\nu:=\frac{1}{2}(r+s-d)$. Since $K \neq \emptyset$ we clearly have $\nu \geq 0$. We claim that

$$
B(z, \nu) \subset K \subset B(z, \nu+\delta) .
$$

To prove the first inclusion it is enough to note that for $w \in B(z, \nu)$

$$
d(w, x) \leq d(w, z)+d(z, x) \leq \nu+\frac{r-s+d}{2}=r
$$

and analogously

$$
d(w, y) \leq d(w, z)+d(z, y) \leq \nu+\frac{s-r+d}{2}=s .
$$

As for the proof of the second inclusion we let $w \in K$ and consider a geodesic triangle with vertices $x_{1}:=x, x_{2}=y, x_{3}=w$ and edges $c_{k \ell}$, where $c_{12}:=c$. Let $a_{1}, a_{2}, a_{3}$ be as in the definition of the $\delta$-thinness. If $d(x, z) \leq a_{1}$ then

$$
d(w, z) \leq d\left(w, c_{13}(d(x, z))\right)+\delta=d(w, x)-d(x, z)+\delta \leq r-\frac{r-s+d}{2+\delta}=\nu+\delta,
$$

whereas if $d(x, z)>a_{1}$ we compute

$$
d(w, z) \leq d\left(w, c_{23}(d(y, z))\right)+\delta=d(w, y)-d(y, z)+\delta \leq s-\frac{s-r+d}{2}+\delta=\nu+\delta .
$$

This establishes the second inclusion in (10) and completes the proof.

\section{Proof of Corollary 1.3}

The following proof uses asymptotic cones. For definitions and basic properties we refer to [2]. We will need the following crucial fact: A geodesic metric space $X$ is Gromov hyperbolic if and only if every asymptotic cone of $X$ is a metric tree, see e.g. [4].

Proof of Corollary 1.3. By Proposition 3.A.1 in [4] it suffices to show that every asymptotic cone of $X$ is a metric tree. Let therefore $\omega$ be a non-principal ultrafilter on $\mathbb{N},\left(x_{n}\right) \subset X$ and $r_{n} \nearrow \infty$ and denote by $X_{\omega}$ the asymptotic cone associated to the sequence $\left(X, \frac{1}{r_{n}} d, x_{n}\right)$ and $\omega$. Clearly, $X_{\omega}$ is geodesic; thus we only need to show that $X_{\omega}$ has property $\left(\diamond^{\prime}\right)$. For this let $\bar{y}=\left(y_{n}\right)$ and $\bar{z}=\left(z_{n}\right)$ be arbitrary points in $X_{\omega}$ and let $r, s>0$ be such that $r+s \geq d_{\omega}(\bar{y}, \bar{z})$. Fix $\varepsilon>0$. By assumption there exist for $\omega$-almost every $n \in \mathbb{N}$ two points $u_{n}, u_{n}^{\prime} \in X$ and $t_{n} \geq 0$ such that

$$
B\left(u_{n}, t_{n}\right) \subset B\left(y_{n},(r+\varepsilon) r_{n}\right) \cap B\left(z_{n},(s+\varepsilon) r_{n}\right) \subset B\left(u_{n}^{\prime}, \lambda t_{n}+\delta\right) .
$$

We have $t_{n} \leq(r+\varepsilon) r_{n}$ from which we conclude that $\bar{t}:=\lim _{\omega} \frac{t_{n}}{r_{n}}$ exists. By the triangle inequality we furthermore have that $\bar{u}:=\left(u_{n}\right)$ and $\bar{u}^{\prime}:=\left(u_{n}^{\prime}\right)$ are elements of $X_{\omega}$. We first claim that

$$
\hat{B}(\bar{u}, \bar{t}-\varepsilon) \subset \hat{B}(\bar{y}, r+\varepsilon) \cap \hat{B}(\bar{z}, s+\varepsilon)
$$


for every $\varepsilon>0$ small enough. Here, $\hat{B}$ denotes a ball in $X_{\omega}$. Let $\bar{v}=\left(v_{n}\right) \in \hat{B}(\bar{u}, \bar{t}-\varepsilon)$ and note that for $\omega$-almost all $n \in \mathbb{N}$ we have $d\left(v_{n}, u_{n}\right) \leq t_{n}$ and therefore, by (11)

$$
\frac{1}{r_{n}} d\left(v_{n}, y_{n}\right) \leq r+\varepsilon \quad \text { and } \quad \frac{1}{r_{n}} d\left(v_{n}, z_{n}\right) \leq s+\varepsilon
$$

for $\omega$-almost all $n \in \mathbb{N}$. This clearly implies that $\bar{v} \in \hat{B}(\bar{y}, r+\varepsilon) \cap \hat{B}(\bar{z}, s+\varepsilon)$. Next, we show that

$$
\hat{B}(\bar{y}, r) \cap \hat{B}(\bar{z}, s) \subset \hat{B}\left(\bar{u}^{\prime}, \lambda \bar{t}\right) .
$$

Indeed, if $\bar{v} \in B(\bar{y}, r) \cap B(\bar{z}, s)$ then $v_{n} \in B\left(y_{n},(r+\varepsilon) r_{n}\right) \cap B\left(z_{n},(s+\varepsilon) r_{n}\right)$ for $\omega$-almost all $n \in \mathbb{N}$ and therefore

$$
d_{\omega}\left(\bar{v}, \bar{u}^{\prime}\right)=\lim _{\omega} \frac{1}{r_{n}} d\left(v_{n}, u_{n}^{\prime}\right) \leq \lim _{\omega} \frac{\lambda t_{n}+\delta}{r_{n}}=\lambda \bar{t},
$$

which proves (12). By Theorem 1.1, which, as we proved, holds when $(\diamond)$ is replaced by $\left(\triangleright^{\prime}\right)$, this is enough to conclude that $X_{\omega}$ is a metric tree.

\section{Appendix: A proof of Theorem 2.3}

A simple proof of the following fact can be found e.g. in [1, Theorem 4.1.6].

Proposition 5.1. Let $X$ be a metric space and $\gamma:(a, b) \rightarrow X$ a Lipschitz curve. Then $\mathrm{md} \varphi_{t}(1)$ exists for almost every $t \in(a, b)$.

Proof of Theorem 2.3. Observe first that if $z \in U$ and $r>0$ and if $v, v^{\prime} \in \mathbb{R}^{k}$ are such that $z+r v, z+r v^{\prime} \in U$ then

$$
\frac{1}{r} d\left(\varphi\left(z+r v^{\prime}\right), \varphi(z)\right)-\varrho \leq \frac{1}{r} d(\varphi(z+r v), \varphi(z)) \leq \frac{1}{r} d\left(\varphi\left(z+r v^{\prime}\right), \varphi(z)\right)+\varrho
$$

where $\varrho:=\operatorname{Lip}(\varphi)\left|v^{\prime}-v\right|$. Moreover, if $z \in U$ and $v, v^{\prime} \in \mathbb{R}^{k}$ are such that $\operatorname{md} \varphi_{z}(v)$ and $\operatorname{md} \varphi_{z}\left(v^{\prime}\right)$ exist then

$$
\left|\operatorname{md} \varphi_{z}(v)-\operatorname{md} \varphi_{z}\left(v^{\prime}\right)\right| \leq \operatorname{Lip}(\varphi)\left|v-v^{\prime}\right| .
$$

We can now easily show that for $\mathcal{L}^{k}$-almost every $z \in U$ the limit $\operatorname{md} \varphi_{z}(v)$ exists for all $v \in \mathbb{R}^{k}$. Indeed, let $\left(v_{i}\right)_{i \in \mathbb{N}} \subset S^{k-1}$ be a countable dense subset. By Proposition 5.1 and Fubini's theorem there exists an $\mathcal{L}^{k}$-negligible set $N \subset U$ such that md $\varphi_{z}\left(v_{i}\right)$ exists for all $i \in \mathbb{N}$ and all $z \in U \backslash N$. Given $v \in S^{k-1}$ arbitrary choose a subsequence $v_{i_{j}}$ of $\left(v_{i}\right)$ which converges to $v$. The existence of md $\varphi_{z}(v)$ now follows immediately from (13) and (14). Moreover, md $\varphi_{z}(s v)$ exists for all $s \geq 0$ and $\operatorname{md} \varphi_{z}(s v)=$ $s \operatorname{md} \varphi_{z}(v)$. Hence, $\operatorname{md} \varphi_{z}(v)$ exists for all $v \in \mathbb{R}^{k}$ and all $z \in U \backslash N$.

We turn to the proof of (4). Denote by $S^{k-1}$ the unit sphere in $\mathbb{R}^{k}$ and by $C\left(S^{k-1}\right)$ the space of continuous real-valued functions on $S^{k-1}$, endowed with the supremum norm. Denote by $D$ the set of points $z \in U$ where $\operatorname{md} \varphi_{z}(v)$ exists for all $v \in \mathbb{R}^{k}$. If $z \in D$ then, by (14), the function $f_{z}: S^{k-1} \rightarrow[0, \infty)$ given by $f_{z}(v):=\operatorname{md} \varphi_{z}(v)$ is $\operatorname{Lip}(\varphi)$-Lipschitz. Furthermore, the map $F: D \rightarrow C\left(S^{k-1}\right)$ given by $F(z):=f_{z}$ is $\mathcal{L}^{k}$-measurable. Since $C\left(S^{k-1}\right)$ is separable, Lusin's Theorem [5, 2.3.5] asserts the existence of compact subsets $K_{i}^{\prime} \subset D$ with $\mathcal{L}^{k}\left(D \backslash \cup K_{i}^{\prime}\right)=0$ and such that $F$ is continuous on each $K_{i}^{\prime}$. In particular, the function $g_{r}(z):=\sup _{|v|=1} \mid \frac{1}{r} d(\varphi(z+$ $r v), \varphi(z))-\operatorname{md} \varphi_{z}(v) \mid$ is continuous on each $K_{i}^{\prime}$, and converges pointwise to 0 as $r \searrow 0$. By Egoroff's Theorem [5, 2.3.7] there exist compact subsets $K_{i}^{j} \subset K_{i}^{\prime}$ with 
$\mathcal{L}^{k}\left(D \backslash \cup_{i, j} K_{i}^{j}\right)=0$ and such that $g_{r}$ converges to 0 uniformly on each $K_{i}^{j}$ as $r \searrow 0$. In particular, for every $\varepsilon>0$ there exists $r\left(K_{i}^{j}, \varepsilon\right)>0$ such that $g_{r}(z) \leq \varepsilon / 2$ and $\left\|\operatorname{md} \varphi_{z}-\operatorname{md} \varphi_{z^{\prime}}\right\|_{\infty} \leq \varepsilon / 2$ whenever $0<r \leq 2 r\left(K_{i}^{j}, \varepsilon\right)$ and $z, z^{\prime} \in K_{i}^{j}$ with $\left|z-z^{\prime}\right| \leq$ $r\left(K_{i}^{j}, \varepsilon\right)$. If $z \in K_{i}^{j}$ and if $v, w \in \mathbb{R}^{k}$ satisfy $|v|,|w| \leq r\left(K_{i}^{j}, \varepsilon\right), v \neq w$, and $z+w \in K_{i}^{j}$ then, setting $r:=|v-w|, z^{\prime}:=z+w$, and $v^{\prime}:=\frac{1}{r}(v-w)$, we obtain

$$
\begin{aligned}
\mid d(\varphi(z+v), & \varphi(z+w))-\operatorname{md} \varphi_{z}(v-w) \mid \\
& \leq r\left|\frac{1}{r} d\left(\varphi\left(z^{\prime}+r v^{\prime}\right), \varphi\left(z^{\prime}\right)\right)-\operatorname{md} \varphi_{z^{\prime}}\left(v^{\prime}\right)\right|+\frac{\varepsilon}{2}|v-w| \\
& \leq r g_{r}\left(z^{\prime}\right)+\frac{\varepsilon}{2}|v-w| \\
& \leq \varepsilon|v-w| .
\end{aligned}
$$

This proves (4) and hence the theorem.

\section{Acknowledgements}

I would like to thank Indira Chatterji for a discussion and Bruce Kleiner for pointing out to me the possibility of proving Corollary 1.3 using our main theorem. I am furthermore indebted to Theo Bühler for many suggestions and comments.

\section{References}

[1] L. Ambrosio and P. Tilli, Topics on analysis in metric spaces, Vol. 25 of Oxford Lecture Series in Mathematics and its Applications, Oxford University Press, Oxford (2004), ISBN 0-19-852938-4.

[2] M. R. Bridson and A. Haefliger, Metric spaces of non-positive curvature, Vol. 319 of Grundlehren der Mathematischen Wissenschaften [Fundamental Principles of Mathematical Sciences], Springer-Verlag, Berlin (1999), ISBN 3-540-64324-9.

[3] I. Chatterji and G. A. Niblo, A characterization of hyperbolic spaces, Groups Geom. Dyn. 1 (2007), no. 3, 281-299.

[4] C. Druţu, Quasi-isometry invariants and asymptotic cones, Internat. J. Algebra Comput. 12 (2002), no. 1-2, 99-135. International Conference on Geometric and Combinatorial Methods in Group Theory and Semigroup Theory (Lincoln, NE, 2000).

[5] H. Federer, Geometric measure theory, Die Grundlehren der mathematischen Wissenschaften, Band 153, Springer-Verlag New York Inc., New York (1969).

[6] É. Ghys and P. de la Harpe, Espaces métriques hyperboliques, in Sur les groupes hyperboliques d'après Mikhael Gromov (Bern, 1988), Vol. 83 of Progr. Math., 27-45, Birkhäuser Boston, Boston, MA (1990).

[7] M. Gromov, Hyperbolic groups, in Essays in group theory, Vol. 8 of Math. Sci. Res. Inst. Publ., 75-263, Springer, New York (1987).

[8] W. B. Johnson, J. Lindenstrauss, D. Preiss, and G. Schechtman, Lipschitz quotients from metric trees and from Banach spaces containing $l_{1}$, J. Funct. Anal. 194 (2002), no. 2, 332-346.

[9] B. Kirchheim, Rectifiable metric spaces: local structure and regularity of the Hausdorff measure, Proc. Amer. Math. Soc. 121 (1994), no. 1, 113-123.

[10] N. J. Korevaar and R. M. Schoen, Sobolev spaces and harmonic maps for metric space targets, Comm. Anal. Geom. 1 (1993), no. 3-4, 561-659.

[11] S. Wenger, Gromov hyperbolic spaces and the sharp isoperimetric constant, Invent. Math. 171 (2008), no. $1,227-255$.

Courant Institute of Mathematical Sciences, 251 Mercer Street, New York, NY 10012

E-mail address: wenger@cims.nyu.edu 